\title{
Pratiques
}

Linguistique, littérature, didactique

181-182 | 2019

Le récit en questions

\section{Récits interactifs et expérience de liberté}

Interactive Narratives and the Experience of Freedom

\section{Dario Compagno}

\section{(2) OpenEdition \\ Journals}

Édition électronique

URL : http://journals.openedition.org/pratiques/5941

DOI : 10.4000/pratiques.5941

ISSN : 2425-2042

Éditeur

Centre de recherche sur les médiations (CREM)

\section{Référence électronique}

Dario Compagno, «Récits interactifs et expérience de liberté », Pratiques [En ligne], 181-182 | 2019, mis en ligne le 30 juin 2019, consulté le 20 juillet 2019. URL : http://journals.openedition.org/ pratiques/5941 ; DOI : 10.4000/pratiques.5941

Ce document a été généré automatiquement le 20 juillet 2019.

(c) Tous droits réservés 


\section{Récits interactifs et expérience de liberté}

Interactive Narratives and the Experience of Freedom

Dario Compagno

\section{Le paradoxe de la liberté ${ }^{1}$}

1 Donner un sens à la vie nous force à nous poser de grandes questions. Est-ce que ce sont les choix qui ont été les miens qui ont fait de moi la personne que je suis ou est-ce plutôt le fruit du hasard? Si j'avais pu prévoir tel évènement, aurais-je eu le courage de faire autrement? Ou au contraire y a-t-il un destin, récit déjà écrit, et quelqu'un qui fait arriver les choses, au-delà de notre responsabilité?

2 Les grandes questions intéressent toujours la philosophie tout entière. La liberté est un problème métaphysique (sommes-nous réellement libres ?), épistémologique (comment concevons-nous notre liberté, toute vraie ou apparente qu'elle soit ?), éthique (qu'est-ce que je devrais faire de ma liberté ?). La liberté est donc également un problème logique ou sémiotique : qu'est-ce que cela signifie être libre? Quel est le sens de nos choix ? Cet aspect est relativement circonscrit, bien qu'il ait des relations avec le concept de liberté dans sa totalité. Le sens de nos actes est un problème de sémiotique narrative et de philosophie analytique de l'action. En effet, c'est un vocabulaire de l'action qui donne un sens à la vie, dont les termes s'appellent choix, conséquence, attente, but, moyen, occasion, hasard, responsabilité, destin, coïncidence, faute, intention, chance. Le sens de la vie dépend d'une manière de distribuer des intentions, d'identifier des résultats prévus ou attendus, de mesurer les efforts pour atteindre des buts, d'évaluer des conséquences.

3 Je voudrais montrer que, grâce aux jeux, et en particulier grâce aux jeux vidéo, nous pouvons faire quelque chose d'autrement totalement inaccessible à la vie quotidienne. Dans les jeux nous pouvons faire l'expérience directe de notre liberté. Pour le dire avec les paroles de J. Henriot, cité par S. Genvo (2013), jouer c'est faire l'exercice du possible. Le jeu est alors une « réalité augmentée » dans un sens fort de l'expression. La réalité est 
grâce aux jeux augmentée parce que les mondes de jeu nous offrent un surplus qualitativement très important. Le jeu ne simule pas la quotidienneté : il va au-delà, parce qu'il nous fait expérimenter la répétition variée et notre intervention dans un monde où les choses ne doivent pas suivre un seul chemin.

Si cela est extrêmement important, c'est parce qu'en effet, dans notre vie nous souffrons d'une grande incapacité, pour le dire à la façon de C.S. Peirce, c'est-à-dire que nous ne faisons jamais l'expérience directe du choix. C'est là un grand paradoxe de l'action humaine, abordé par maints philosophes. Réfléchissons : toute action est faite une et une seule fois. Nous pouvons réaliser plusieurs fois des actions du même type (nous préparons le café chaque matin), mais chaque action concrète, chaque occurrence du type, est unique. Dans la vie quotidienne nous ne pouvons pas rembobiner la bande et vivre une deuxième fois un certain moment. Dans un sens intuitif, être libre signifie que dans une certaine situation nous avons la pleine et entière responsabilité de ce qui adviendra. Dans des termes plus précis, nous aurions pu faire autrement - première condition de l'action libre, l'ouverture - et nous avons eu un rôle déterminant dans ce que nous avons effectivement choisi - deuxième condition de l'action libre, l'intervention (voir De Caro, 2009). Un agent, au sens plein du terme, a la capacité de déterminer un cours d'action parmi d'autres qui sont tous effectivement accessibles à lui avant le choix.

Mais comment vérifier empiriquement ces deux conditions, ouverture et intervention? Comment vérifier si nous aurions pu faire autrement? Pour le savoir avec certitude, nous devrions revivre le moment et prendre une voie différente. Un peu comme cela arrive aux protagonistes de films tels que Funny Games (M. Haneke, 1997), Cours, Lola, cours (T. Tykwer, 1998) ou Donnie Darko (R. Kelly, 2001). Malheureusement, nous n'avons pas cette capacité dans la vraie vie. Nous ne savons pas si la vie quotidienne suit effectivement un modèle modal, au sens de S. Kripke (voir Goldblatt, $1987 ;$ Eco, 1985), où plusieurs futurs possibles sont ouverts avant d'en prendre un. Il n'y a pas d'alternatives visibles. L'impression de liberté qui est la nôtre est en effet juste un sentiment immédiat. A. Schopenhauer écrivait dans Sur la liberté de la volonté humaine (1839) que le processus de décision n'est jamais conscient ; nous savons juste quel est le choix que nous avons fait, à posteriori, selon ce que notre volonté nous a fait faire. Le choix transcende la conscience, qui se limite à en prendre acte (voir Compagno, 2008).

Il semble absurde qu'alors le jeu ait ce pouvoir incroyable de nous faire faire l'expérience directe de la liberté d'action. Jouer est une expérience très particulière, ordinaire et infantile, qui nous permet de donner un contenu à l'idée de choix. Les jeux vidéo sont des objets sémiotiques qui nous permettent de compenser notre incapacité épistémologique, et donc de vivre des choix que nous savons être vraiment à nous, parce qu'ils sont reconductibles. Cela montre que l'objet jeu vidéo est une «machine expérimentale » en puissance pour le raisonnement philosophique, comme le suggère S. Gualeni (2015).

\section{Deux modèles de l'action}

7 L'héritage des philosophies modernes de l'action a été poursuivi au $\mathrm{xx}^{\mathrm{e}}$ siècle en Angleterre et en France sous deux formes proches, mais finalement incompatibles, adaptées à deux régimes interprétatifs différents, c'est-à-dire à deux manières différentes d'envisager le fonctionnement des signes (Compagno, 2009). D'un côté nous avons la vie quotidienne, où habitent les personnes que nous connaissons et avec qui nous interagissons plus ou moins directement. Quand nous parlons avec quelqu'un, nous le 
concevons comme une personne responsable de ses choix, en tant qu'agent donc, capable de réaliser plusieurs stratégies dans le jeu de langage. D'un autre côté, il y a le roman, c'est-à-dire le récit prototypique. Quand nous lisons des actions des personnages nous savons qu'il y a quelqu'un d'autre derrière tout ce qui est raconté. Les actions sont présentées en accord avec une stratégie unitaire qui structure le roman, dirigée par un auteur caché et effectif, à ne pas confondre avec les personnages visibles et fictifs (Compagno, 2012).

8 La sémiotique, d'un côté, propose une forme de description capable d'interpréter les romans, et le modèle développé par A. J. Greimas (1970) en montre exemplairement les points de force et de faiblesse. Quand nous faisons d'un roman un texte, nous reconnaissons une instance unique derrière tout ce qui se passe dans le monde fictionnel. Faire ce pas nous permet de mieux comprendre le sens des actions racontées, qui ont toujours un rôle relationnel, et sont toutes orientées vers la même conclusion « logique " et «morale » du récit. Les personnages ou acteurs d'une histoire ne peuvent que suivre le plan d'un metteur en scène. Ils ne font que ce qu'on veut leur faire faire. D'ailleurs, déjà Aristote dans la Poétique écrivait que l'action dramatique n'est pas une suite d'épisodes mais le lien qui les structure en unité. Cette leçon, assimilée par le formalisme soviétique, est l'essence d'une approche structurale du récit où le tout vaut bien plus que la somme de ses parties.

De l'autre côté de la Manche, d'autres formes de description ont été élaborées pour décrire notre manière de donner un sens à la vie quotidienne. E. Anscombe (2002) a en particulier développé un modèle qui présuppose et permet de calculer des intentions derrière les actions. Les intentions se devinent à partir des actes réalisés, ne se trouvant aucunement cachées dans la tête des gens; elles sont le résultat public - sui-référentiel dirait-on avec les mots d'É. Benveniste - du comportement finalisé et spécialement de l'utilisation du langage. Sans intention, les actes ne seraient que des comportements automatiques que nous ne pouvons pas interpréter de la même manière : tousser n'est pas agir, sauf si j'y vois une intention, et je pense que mon voisin, en toussant, veut me dire quelque chose avec discrétion. Pour E. Anscombe, nous devons penser aux autres comme à des agents libres, sinon notre grammaire de l'action ne marche plus.

Or les théories structurales et analytiques de l'action sont profondément incompatibles. La vie quotidienne n'est pas un roman, et vice versa; chercher alors à décrire les agents libres comme s'ils étaient des personnages et ceux-ci comme s'ils pouvaient faire autrement ne peut que conduire à un échec. Pensons à ce qui pourrait arriver si nous appliquions tel quel le modèle analytique d'E. Anscombe à un roman. Si nous faisions comme si les personnages fictifs sont des agents, alors nous n'arrivons plus à comprendre la nature du roman, spécialement le fait que le tout du récit a un sens ultérieur qui est plus que la somme de ses parties. Comment saisir le fait que le sens d'un roman n'est pas la succession d'épisodes mais une unité à lire à rebours? Que deviennent dans cette perspective l'auteur transcendant et sa contrepartie le destin immanent au récit (voir cidessous) ? Vu de près, le modèle analytique ne marche pas trop bien pour comprendre la fiction.

11 On prend des risques symétriques à vouloir appliquer le modèle structural à la vie quotidienne, c'est-à-dire aux pratiques sociales. Si je pensais que les actions de mes collègues présents à un congrès étaient guidées par quelqu'un, et qu'il y avait des fils invisibles qui les constituaient en unité, visant un seul sens global, je serais pris pour un vrai croyant, ou plus probablement pour un paranoïaque. Qui serait, en effet, l'auteur des 
actions humaines? Le modèle structural conduit à des descriptions qui ne sont pas adéquates au régime interprétatif de la vie quotidienne parce qu'il ne prend pas en compte la responsabilité individuelle, mais cherche au contraire à tout ramener à une structure déterministe ou à un plan supra-individuel.

\section{Le hasard, seuil entre vie et récit}

Pour montrer plus exactement la spécificité du récit par rapport à la vie quotidienne, prenons l'exemple de La Mort d'Ivan Ilitch de L. Tolstoï. Ce récit est structurellement très élégant parce que tout en lui se tient à partir d'une petite clé de voûte, d'un moment crucial qui porte tout le reste. La morale du récit ne repose que sur deux lignes glissées dans le texte sans préavis. En simplifiant à l'extrême, La Mort d'Ivan Ilitch (1886) concerne la vie d'un magistrat, heureux de sa carrière jusqu'au moment où, pendant qu'il meublait son nouvel appartement, il faillit tomber d'un escalier :

\section{Un jour, en montant sur une échelle pour expliquer au tapissier, qui ne comprenait pas, comment il voulait draper les rideaux, il fit un faux pas et tomba ; mais comme il était adroit et vigoureux, il se retint et se cogna seulement le côté à l'espagnolette. Il en souffrit pendant quelques jours, puis la douleur disparut. (Tolstoï, 1912, p. 36.)}

Dès la première lecture, il est évident que ces deux lignes vont jouer un rôle fondamental, et que les choses vont commencer à aller de pire en pire. Il s'agit d'une annonce ou d'un indice, élément du code herméneutique décrit par R. Barthes (1970). Un évènement qui relève du hasard - mieux, qui devrait relever du hasard s'il se passait dans la vraie vie montre tout de suite la main de l'auteur. Cela parce que, tout simplement, dans les récits $i l$ n'y a pas de hasard, tout est coïncidence intentionnelle. Rien ne se passe sans que l'auteur le désire. Comme l'écrivait encore R. Barthes (1966), le récit est le langage du destin. Et c'est alors bien dans le récit d'évènements fortuits que l'on peut voir les choix de l'auteur. Il vaut mieux chercher l'auteur là où les simulacres sont le plus efficacement dissimulés parce qu'impersonnels. Si je ne peux rapporter une décision qu'à l'auteur, qu'à L. Tolstoï en personne, sans aucune hésitation, c'est justement celle derrière cette chute, à l'apparence aléatoire et anodine et en réalité pleine de conséquences. J.-P. Sartre (1948, p.60-61) l'écrivait avec une clarté exemplaire. À la différence d'un agent, toujours incertain des vicissitudes de la vie,

le lecteur, au contraire, progresse dans la sécurité. Aussi loin qu'il puisse aller, l'auteur est allé plus loin que lui. Quels que soient les rapprochements qu'il établisse entre les différentes parties du livre - entre les chapitres ou entre les mots - il possède une garantie : c'est qu'ils ont été expressément voulus. Il peut même, comme dit Descartes, feindre qu'il y ait un ordre secret entre des parties qui ne semblent point avoir de rapports entre elles ; le créateur l'a précédé dans cette voie et les plus beaux désordres sont effets de l'art, c'est-à-dire ordre encore [...] L'arbre et le ciel, dans la nature, ne s'harmonisent que par hasard; si, au contraire, dans le roman, les héros se trouvent dans cette tour, dans cette prison, s'ils se promènent dans ce jardin, il s'agit à la fois de la restitution de séries causales indépendantes [...] et de l'expression d'une finalité plus profonde, car le parc n'est venu à l'existence que pour s'harmoniser avec un certain état d'âme [...] Ici c'est la causalité qui est l'apparence et qu'on pourrait nommer «causalité sans cause », et c'est la finalité qui est la réalité profonde.

Dans le récit, le hasard devient un personnage, probablement le plus important et sousestimé. C'est alors justement le hasard qui constitue le seuil net entre action quotidienne et 
action racontée, entre le domaine propre du modèle analytique et celui du modèle structural. Le modèle analytique, forward looking, ne peut pas, d'aucune manière, rendre compte des coïncidences et de leur rôle, qui vont au-delà des intentions individuelles les liant au plan général du récit. De même, le modèle structural, backward looking, peine à rendre compte de l'existence de vrais hasards dans la vie quotidienne, d'un hasard aveugle qui ne structure rien et qui n'a aucun sens caché ${ }^{2}$.

\section{Le jeu comme volonté dans la représentation}

15 Jusqu'ici, nous avons vu rapidement que la compréhension des deux régimes interprétatifs différents de la vie quotidienne et du récit, nécessite d'avoir recours à deux modèles mutuellement exclusifs. Il n'y a pas de perspective unitaire capable de bien prendre en compte à la fois les actions quotidiennes et les actions racontées. Il s'agit en fait de deux objets très proches et en même temps séparés par un seuil non banal - la présence ou l'absence de vrai hasard. En conséquence, la grammaire des actions racontées est effectivement spéciale, où tout ce qui advient est intentionnel à un certain niveau de description ${ }^{3}$.

L'incompatibilité des deux modèles devient mieux visible si l'on tente de les appliquer à la description de l'action interactive, telle qu'on la trouve par exemple dans les jeux vidéo. Un récit interactif nait quand au moins un choix qui détermine le développement du monde de jeu est dans les mains du joueur (Compagno, 2006). En conséquence, le joueur a un accès, même très limité et encadré en amont, à la transcendance de l'đEuvre, c'est-àdire à la série de choix responsables pour la réalisation d'une variante contingente, d'un texte tel qu'il existe effectivement devant nous (Genette, 1994). Aucune interactivité n'est possible sans une interférence entre l'immanence du monde du jeu et les actions externes des joueurs. On voit que les actions des personnages des récits interactifs contrôlés par des joueurs manifestent bien des vraies intentions, telles qu'on les trouve dans la vie quotidienne. En même temps, ces intentions s'exercent entre les limites d'un plan global qui est absent dans la vraie vie (en dehors évidemment d'une interprétation religieuse de l'existence). De façon paradoxale, l'action réelle du joueur va faire partie d'un récit fictionnel et cette fiction va lui donner un sens réel.

Afin de rendre compte de l'action interactive nous avons alors besoin d'une extension de la narratologie structurale, capable de rendre compte premièrement d'un parallélisme entre fiction et réalité, et deuxièmement de l'incidence entre ces deux plans.

\section{Parallélisme}

La théorie d'A. Schopenhauer nous est utile d'abord parce qu'elle permet de coupler de manière très élégante les actions représentées, par exemple dans le monde de pixels d'un jeu vidéo, avec les actions quotidiennes des joueurs. A. Schopenhauer écrivait dans Le Monde comme volonté et représentation (1818-1819) que le phénomène, c'est-à-dire le monde que nous percevons, est immanent à la conscience (le monde tel que nous le connaissons n'existe que pour nous). Surtout, tout ce qui se passe dans le phénomène est nécessité : toute action ou évènement suit le principe de causalité ou de raison suffisante ( $L a$ Quadruple racine du principe de raison suffisante, 1813). Tout s'enchaine et tout se tient, sans exception, depuis la nuit des temps et pour toujours. Bien sûr, d'autres philosophes avaient déjà décrit le monde sous la forme de l'immanence, du déterminisme et de la 
nécessité - nous pensons par exemple à Spinoza. Mais A. Schopenhauer, suivant son maitre I. Kant, ajoute que ce déterminisme ne vaut que pour l'apparence de la vie, pour le phénomène, parce que c'est notre cerveau qui met en relation les évènements en utilisant en guise de ciment le principe de la raison suffisante.

Or, l'originalité de la pensée d'A. Schopenhauer par rapport à I. Kant, consiste à dire que derrière l'immanence du phénomène, de ce monde construit par notre cerveau, il y a aussi un responsable de tout ce qui se passe, c'est-à-dire une volonté transcendantale. La volonté est libre parce qu'au-delà du phénomène le principe de raison suffisante ne vaut plus. Cette volonté est unique, unitaire, elle est la même pour toutes les personnes du monde ; plus que cela, même les évènements naturels manifestent cette volonté. Donc si un volcan " décide » d'entrer en éruption, deux descriptions sont possibles et les deux sont également correctes. D'abord, le volcan est entré en éruption parce que des causes physiques ont déterminé cet évènement, affaire de volcanologie. Cause et effet, on reste dans la description immanente du phénomène, pour laquelle il n'y a pas de place pour la liberté. En même temps, si je dis que le volcan a "décidé » d'entrer en éruption, c'est parce que vraiment une décision a été prise, par la volonté transcendantale qui se manifeste à travers le volcan.

Le monde est un roman, finalement, ou mieux une pièce de théâtre : son sens dépend bien sûr des acteurs sur scène, mais il ne faut pas oublier les choix du réalisateur, même s'ils ne se manifestent que très discrètement. La volonté cachée est même la seule chose qui compte vraiment pour le philosophe allemand. Comme l'écrivait d'ailleurs J.-P. Sartre (1948, p. 63) au sujet du récit : «Ainsi à travers la causalité phénoménale, notre regard atteint la finalité, comme la structure profonde de l'objet et, au-delà de la finalité, il atteint la liberté humaine comme sa source et son fondement originel. »

21 Avec un peu d'imagination on peut alors essayer d'appliquer cette vision du monde à la lettre aux actions racontées. En effet, d'une première perspective immanente, les récits sont des petits mondes, bien fermés et détachés du monde réel où on les a produits. Tout ce qui se passe dans un récit se tient, post hoc propter hoc, les actions et les évènements s'enchainent suivant une nécessité narrative (Barthes, 1966 ; Ricœur, 1984 ; Dubied, 2000) et tout contribue à un sens global. Or, suivant A. Schopenhauer, il y a en parallèle une transcendance à prendre en compte, une volonté unique qui fait arriver tout ce qui se passe dans le monde du récit. Toute action racontée, mais aussi tout évènement représenté comme fortuit, est sous la responsabilité d'une seule finalité transcendantale. Cette volonté, depuis toujours, on l'appelle l'auteur. Le monde du récit est une représentation autonome et suit des règles qui lui sont propres, mais il ne faut pas oublier la volonté qui décide ailleurs réellement de ce qui va se passer (voir Compagno, 2008, 2009, 2012).

Si l'on se limitait à analyser des récits traditionnels, comme la nouvelle de L. Tolstoï citée ci-dessus (§3), on pourrait avoir l'impression que cette mise à jour critique n'est pas vraiment nécessaire. On pourrait dire que bien évidemment il n'y a pas d'histoire sans quelqu'un qui la raconte, mais que cette instance racontante, externe au monde textuel, est finalement inanalysable et en plus inutile pour l'interprétation. Il s'agit de la célèbre thèse de W. K. Wimsatt et M.C. Beardsley (1946): l'intention de l'auteur est inconnaissable et inintéressante. L'immanence suffirait à elle-même. Cette perspective théorique a montré ses insuffisances déjà pour les récits traditionnels (surtout s'ils sont ouverts au sens d'U. Eco, voir ci-dessous, $\$ 5$ ); mais dès que l'on approche un récit interactif où le joueur participe activement de la volonté transcendantale, en faisant des 
choix qui déterminent l'évolution du monde fictionnel, une description purement immanente devient tout simplement impossible. Pour comprendre les jeux vidéo, et l'expérience de liberté qu'ils permettent, nous avons besoin de penser aux choix réels qui déterminent ce qui se passe dans le monde du jeu. Immanence et transcendance, ensemble, sont nécessaires pour l'analyse de l'interactivité.

\section{Incidence}

Toujours grâce à A. Schopenhauer nous avons les moyens de prendre en compte l'intégration d'une composante transcendante (ou si l'on veut pragmatique) à l'analyse. Cela parce qu'A. Schopenhauer ne s'est pas limité à souligner la transcendance parallèle à l'immanence du phénomène. Il a réalisé un deuxième pas théorique fondamental, en montrant le lieu où l'immanence et la transcendance se touchent et se relient l'une à l'autre. En effet, il y a une manifestation de la volonté transcendantale dans le phénomène auquel j'ai un accès direct : c'est quand la volonté devient la mienne. Dès que mon corps est concerné j'ai le sentiment immédiat d'agir, parce que la volonté me prend comme son véhicule privilégié. Il y a donc un «trou » dans l'immanence du phénomène, et il s'agit de moi-même; j'accède directement au noumène quand il me pousse à agir cela correspond bien à ce qu'U. Eco appelle le Qualcosa-che-mi-prende- $a$-calci'

Les philosophies orientales, desquelles A. Schopenhauer s'est beaucoup inspiré, mentionnent des avatars, c'est-à-dire des corps à travers lesquels les divinités agissent dans le monde. Les avatars permettent à la transcendance, aux dieux, d'agir dans l'immanence de la vie quotidienne, prenant un corps. A. Schopenhauer récupère cette image, pour dire que les personnes se sentent libres, parce que la volonté qui les manipule, cette sorte de volonté divine, est vécue comme propre et émanant de soimême, du corps propre. Donc pour A. Schopenhauer mon corps est l'avatar de la volonté dans l'immanence du phénomène; c'est dans mon corps que la volonté, l'auteur du monde, manifeste des choix que j'avertis comme miens. La pertinence de ces considérations pour comprendre les jeux vidéo ne demande pas beaucoup de fantaisie.

Prenons un exemple célèbre de jeu vidéo, The Legend of Zelda (1986). Il s'agit d'un jeu de rôle à la japonaise à thème fantastique. Le protagoniste de ce jeu s'appelle Link, mot qui signifie «lien » en anglais. Link est l'avatar du joueur pendant toute la partie. C'est à travers Link que nos choix peuvent contribuer à déterminer le déroulement de l'histoire racontée. Sans Link, sans lien, donc avec la transcendance, tous les choix qui déterminent ce qui se passe dans le monde de jeu seraient sous la responsabilité des game designers. On serait en train de regarder un dessin animé. Mais justement non, dans The Legend of Zelda nous joueurs pouvons agir, agir réellement, dans le sens de la philosophie analytique de l'action. Nous pouvons faire des choix parmi des options ouvertes. Link est peut-être un personnage de papier ou de pixels, mais grâce à lui nous pouvons manifester des vrais choix dans l'immanence du jeu. Il y a un "trou » qui permet l'incidence entre deux mondes, volonté et représentation, et ce « trou », A. Schopenhauer le savait bien, est bien l'avatar. L'avatar est un corps perçu dans l'immanence du phénomène et habité par un au-delà (Compagno, 2008).

En analysant les jeux vidéo, le fonctionnement de l'appareil formel de l'énonciation devient alors très intéressant, et le mot «je» en particulier montre une duplicité caractéristique. «J'ai tué le dragon » fait référence en même temps à l'avatar qui a, dans son monde fictif, tué le dragon et à moi jouer que j'ai, dans mon monde réel, gagné la 
partie en tuant le dragon (voir aussi Genvo, 2009). La description «j'ai appuyé sur un bouton » ne serait pas suffisante pour rendre compte de l'action du joueur. En appuyant sur un bouton, je peux appeler l'ascenseur tout comme déclencher une guerre nucléaire, et il s'agit de deux actions quand même assez différentes. En effet, comprendre l'action réelle du joueur demande des descriptions qui font également référence à l'action de l'avatar dans le monde fictionnel. S. Genvo (2013) écrit à ce propos :

Comme le disent les amateurs de jeu d'échecs, il ne s'agit pas uniquement de "pousser du bois». Pour entrer véritablement dans le jeu, le joueur doit faire comme si le plateau et les pièces qui se présentent à lui ne se résumaient pas uniquement à ce qu'ils sont, mais sans prendre pour autant le simulacre pour la réalité ; il doit entrer dans un univers de sens singulier : il va transposer les choses du monde où il vit dans un ordre nouveau, régi à la fois par des règles de la vie courante et par des règles spécifiques qui n'y ont pas habituellement cours.

\section{Interagir c'est fermer une ouverture} déclinaisons françaises ou anglaises. Toute action de jeu est double: elle advient dans l'immanence du monde fictif, au-delà de l'écran, et en même temps dans la transcendance du monde réel, en deçà de l'écran 5 . S. Genvo (2013) écrit que pour rendre compte des jeux vidéo il faut les penser sous les deux catégories de game et de play. Donc en étudiant d'abord leurs règles immanentes, le sens des coups par rapport à la planche, et ensuite également les actions des joueurs, qui ont leurs propres normes de l'action ordinaire. Prenons comme exemple l'interdiction de tricher, valable plus ou moins strictement selon le contexte de la partie. "J'ai triché » est typiquement une description qui ne vaut que pour le joueur, et pas pour les personnages. Moi, je triche, mon avatar, il tue le dragon. Cependant, on ne peut donner un sens à l'expression "j'ai triché » qu'en connaissant les règles immanentes du game, qu'on serait censé respecter. On doit savoir ce qui est prévu et ce qui ne l'est pas, on doit donc saisir le joueur modèle du jeu, pour ensuite tenter de se jouer de lui. S. Genvo (2013, § 16) reprend le concept de joueur modèle de la sémiotique d'U. Eco ${ }^{6}$ et écrit :

Elle [une structure de jeu] dessine en somme un certain « joueur-modèle », qui n'est pas nécessairement le joueur empirique mais qui est en tout cas la stratégie interprétative que met en forme la structure de jeu pour constituer l'ensemble de ses interprétations légitimables. Le joueur-modèle témoigne du contexte pragmatique pour lequel la structure de jeu a été initialement conçue, ce qui n'empêche aucunement les réappropriations.

Le joueur modèle combine des prérogatives du lecteur modèle et de l'auteur modèle, tels qu'ils sont conçus par U. Eco (1979, voir Compagno, 2006). Le joueur modèle est en effet un modèle de comportement à respecter, une interface pour bien interpréter le jeu et pouvoir en saisir un sens légitimable. En même temps, le lecteur devient joueur s'il a accès à au moins un choix qui détermine le développement du monde du jeu, il participe donc à la constitution de l'auteur modèle, de la stratégie responsable du texte. Dans un texte traditionnel comme un roman, l'auteur modèle est totalement inaccessible au lecteur; le seul à prendre des vraies décisions est l'auteur. Or, cela est vrai en général, sauf dans les ceuvres ouvertes. Le concept d'ouverture est la clé de voûte de toute la pensée d'U. Eco, et c'est l'ouverture qui va nous permettre de relier game et play, et plus généralement, les actions racontées et les actions ordinaires.

Pratiques, $181-182$ | 2019 
29 En 1962, U. Eco publie en Italie L'CEuvre ouverte. Ce livre cause un petit tremblement de terre intellectuel et ouvre la période post-structurale en Europe. Quand C. Lévi-Strauss lit ce livre, il répond que ce n'est pas possible, les œuvres sont fermées, leur sens est purement immanent; les œuvres, dit C. Lévi-Strauss, sont comme des cristaux, où il n'y a pas de place pour l'indétermination ${ }^{7}$. Cela parce qu'U. Eco proposait d'analyser des œuvres d'art contemporaines, ou des objets de communication nouveaux à l'époque, comme le direct télévisé, en valorisant leur ouverture : des sacs où soit le hasard, le vrai hasard, est à l'œuvre, soit le lecteur peut prendre des décisions, des vraies décisions. Voilà que les deux impensables de la théorie structurale montrent leur tête - hasard et intention, qui ne devraient exister dans le récit (voir ci-dessus, § 3), ne peuvent pas être mis entre parenthèses.

30 Regarder un match de football n'est pas faire la même expérience que lire un roman, parce que personne ne sait qui gagnera, sauf bien sûr si l'une des équipes a acheté le match en avance. Le réalisateur d'un direct à la télévision doit gérer le hasard et les intentions des agents participants, c'est-à-dire qu'il doit mettre en récit une matière qui ne dépend pas de sa seule volonté. La volonté transcendantale se trouve à devoir négocier avec ses manifestations, un peu comme cela arrive dans Six personnages en quête d'auteur de L. Pirandello (1921). Dans les œuvres ouvertes les plus traditionnelles et réussies, l'auteur laisse un espace vide significatif qui sera comblé par l'imagination du lecteur, et grâce à ce vide l'auteur lui laisse donc un espace de liberté. Si moi, auteur, je ne choisis pas, alors toi, lecteur, tu pourras imaginer ce que tu préfères. On pourrait citer comme exemple une autre pièce de L. Pirandello, À chacun sa vérité (1917).

31 Les œuvres ouvertes procurent aux lecteurs ou aux spectateurs un sentiment de vertige. On voit soudainement qu'il y avait un auteur, parce qu'il ne fait pas ce qu'il était censé faire : choisir. Le lecteur ou spectateur a alors de la place, il doit imaginer pour soi-même, et souvent il n'a pas l'habitude de cela et doit adapter son mode de lecture.

Il reste que les œuvres ouvertes les plus traditionnelles, comme celles citées ci-dessus, ne demandent quand même pas d'agir matériellement, c'est-à-dire de fermer l'ouverture auctoriale. Le monde du récit reste ouvert et on ne peut rien faire pour le refermer, on ne peut pas faire un choix là où l'auteur a décidé de ne pas choisir. Si l'on regarde un film open ended comme Inception (C. Nolan, 2010), on ne peut pas décider une fois pour toutes si la dernière scène est un rêve ou si c'est la réalité : le doute va rester pour toujours. Les œuvres ouvertes en général ne sont donc pas interactives. Or, il faut ajouter qu'U. Eco avait déjà compris, bien avant la naissance des jeux vidéo, l'importance de l'interactivité. $\mathrm{Au}$ point qu'il appelait en 1962 "œuvres en mouvement» une famille bien spéciale d'œuvres ouvertes. Dans les œuvres en mouvement c'est l'action réelle du lecteur ou du spectateur qui va déterminer ce qui se passe dans l'histoire racontée ou dans sa mise en récit ${ }^{8}$. Comme exemple célèbre, précédant l'époque vidéoludique, on pourrait citer Marelle (1966) de J. Cortázar.

33 Il y a donc des objets qui ne nous offrent pas seulement une ouverture, c'est-à-dire un éventail d'alternatives possibles, mais aussi des moyens techniques pour agir, pour choisir et déterminer une de ces possibilités. À partir de la sémiotique d'U. Eco, on peut alors donner une définition minimale de l'interactivité : l'interactivité, c'est une ouverture qui demande et permet d'être fermée (Compagno, 2006). Pour le dire autrement, il y a une immanence ouverte, "trouée ", non nécessitée, et un acte transcendant qui va fermer cette indétermination, en faisant un vrai, libre, choix. Accidentellement, cela revient aux deux conditions nécessaires de l'action libre, ouverture et intervention, telles qu'elles ont 
été définies par la philosophie analytique (voir De Caro, 2009 et ci-dessus, § 1). Le futur d'un agent doit être ouvert à plusieurs chemins et l'agent doit être capable de déterminer ce qui va se passer. L'interactivité est donc point par point le correspondant sémiotique de l'action libre.

\section{Conclusion}

Si je joue à Super Mario Bros (1985), Mario ne peut pas bouger sans moi. Je dois imaginer des déroulements possibles dans le monde fictionnel et faire des choix réels pour actualiser un «bon » futur. Magie des jeux vidéo, si je joue à Super Mario Bros, et si je fais un mauvais choix, si je tombe par exemple dans un gouffre, je peux rembobiner et essayer à nouveau. Mon acte était vraiment libre, j'en suis sûr maintenant, parce que j'ai pu le refaire, et je sais donc ce qui arrive si je fais autrement. Le jeu vidéo rend visible la virtualité des possibles.

Est-ce qu'il en va de même dans la vie quotidienne ? Aurais-je pu éviter tel gouffre ? On ne le saura jamais, on ne saura jamais si j'étais obligé, destiné, à suivre un parcours déterminé. On aurait beau passer des heures à spéculer sur les conséquences possibles de nos actions passées. Hillary Clinton aurait-elle pu gagner? Adolf Hitler aurait-il unifié l'Europe? Les philosophes analytiques appellent contrefactualité ce type de raisonnement un peu inconclusif (Lewis, 1973). Et P. Ricœur (1984) l'a d'ailleurs écrit clairement, l'histoire des historiens est un récit, un récit traditionnel : le hasard et les intentions y meurent, et l'historien vise juste à bien raconter ce qui s'est passé, sans chercher des alternatives. L'objet jeu vidéo est alors résolutif parce qu'on peut tout savoir si l'on analyse les actions en jeu; l'épaisseur modale de ce qui ne s'est pas avéré est encore accessible et actualisable. Analyser un jeu vidéo demande alors de développer une grammaire de l'action capable de décrire comment un sens se produit dans l'interaction entre, d'un côté, une histoire pré-formée et, de l'autre, la vraie responsabilité du joueur.

U. Eco (1999) a écrit que les romans nous enseignent l'inéluctabilité - à mourir - parce que nous ne pouvons pas changer leur fin. On pourrait ajouter que les jeux vidéo nous enseignent bien d'autres choses, la liberté et la responsabilité - à vivre - parce que c'est à nous de fermer leur ouverture, de prendre la parole après le silence.

\section{BIBLIOGRAPHIE}

AARSETH, E. (1997). Cybertext. Perspectives on Ergodic Literature. Baltimore/London : Johns Hopkins University Press.

ANSCOMBE, E. (2002) [1957]. L'Intention. Trad. de l'anglais par M. Maurice et C. Michon. Paris : Gallimard.

BARTHES, R. (1966). «Introduction à l'analyse structurale du récit ». Communication 8, p. 1-27. En ligne : https://www.persee.fr/doc/comm_0588-8018_1966_num_8_1_1113. 
BARTHES, R. (1970). S/Z. Essais. Paris : Éditions du Seuil.

BRONNER, G. (2007). Coïncidences. Paris : Vuibert.

COMPAGNO, D. (2006). « Donnie Darko : tra apertura e ipertestualità ». Ocula 7. En ligne : https:// www.ocula.it/files/OCULA-7-COMPAGNO-Donnie-darko-tra-apertura-e-ipertestualita.pdf.

COMPAGNo, D. (2008). « I am Link's Transcendental Will ». In : Cuddy, L. (dir.), The Legend of Zelda and Philosophy. Chicago : Open Court, p. 185-202.

COMPAGNO, D. (2009). «Fate or Agency? ». Lexia 3-4, p. 231-244.

COMPAGNo, D. (2012). «Double énonciation d'auteur et de narrateur ». In : Carel, M. (dir.),

Argumentation et polyphonie. Paris : L'Harmattan, p. 105-144.

CORTÁZAR, J. (1966). [1963]. Marelle. Trad. de l'espagnol par L. Guille et F. Rosset. Paris : Gallimard. DE CARO, M. (2009). Il libero arbitrio. Una introduzione. Roma/Bari : Laterza.

DUBIED, A. (2000). « Une définition du récit après Paul Ricœur ». Communication 19 (2), p. 45-66. En ligne : https://journals.openedition.org/communication/6312.

ECO, U. (1965) [1962]. L'CEuvre ouverte. Trad. de l'italien par C. Roux de Bézieux. Paris : Éditions du Seuil.

ECO, U. (1985) [1979]. Lector in fabula. Le rôle du lecteur ou La coopération interprétative dans les textes narratifs. Trad. de l'italien par M. Bouzaher. Paris : Grasset.

ECO, U. (1999). «Storie già fatte e storie da fare ». In : La bustina di Minerva. Milan : Bompiani, p. 193-194.

ECO, U. (2001) [1997]. Kant et l'ornithorynque. Trad. de l'italien par J. Gayrard. Paris : Grasset.

GENETTE, G. (1994). L'Euvre de l'art. Immanence et transcendance. Paris : Éditions du Seuil.

GENVO, S. (2009). « Le rôle de l'avatar dans la jouabilité d'une structure de jeu vidéo ». Adolescence 69 (3), p. 645-655. En ligne : https://www.cairn.info/revue-adolescence1-2009-3-page-645.htm.

GENVO, S. (2013). « Penser les phénomènes de ludicisation à partir de Jacques Henriot ». Sciences du jeu 1. En ligne $:$ https://journals.openedition.org/sdj/251.

GOLDBLATT, R. (1987). Logics of Time and Computation. Stanford : CSLI.

GREIMAS, A. J. (1970). Du sens. Paris : Éditions du Seuil.

GUALENI, S. (2015). Virtual Worlds as Philosophical Tools. London : Palgrave MacMillan.

LEWIS, D. (1973). Counterfactuals. Oxford : Blackwell.

MAIETTI, M. (2004). Semiotica dei videogiochi. Milan : Unicopli.

MURRAY, J. (1997). Hamlet on the Holodeck. The Future of Narrative in Cyberspace. Cambridge (MA) : MIT Press.

RIC@UR, P. (1984). Temps et récit. T. 2. Paris : Éditions du Seuil.

RIC@UR, P. (1988). « L'identité narrative ». Esprit 140-141, p. 295-304.

SARTRE, J.-P. (1948). Qu'est-ce que la littérature? Paris : Gallimard.

SCHOPENHAUER, A. (1966) [1818-1819]. Le Monde comme volonté et comme représentation. Trad. de l'allemand par A. Burdeau. Paris : Presses universitaires de France. 
SCHOPENHAUER, A. (1998) [1813]. De la quadruple racine du principe de raison suffisante. Trad. de l'allemand par F.-X. Chenet. Paris : Vrin.

SCHOPENHAUER, A. (2011) [1839]. Sur la liberté de la volonté humaine. Trad. de l'allemand par E. Osier. Paris : Hermann.

SEARLE, J. (1975). « The Logical Status of Fictional Discourse ». New Literary History 6 (2), p. 319-332. TOLSTOÏ, L. (1912) [1886]. La Mort d'Ivan Ilitch. In : CEuvres complètes. Trad. du russe par J. W.

Bienstock. Paris : Stock, vol. 27, p. 3-105, 1912.

WimsatT, W. K. \& BEARDSLey, M. C. (1946). « The Intentional Fallacy ». The Sewanee Review 54 (3), p. $468-488$

\section{NOTES}

1. Ce texte est une synthèse de certains travaux conduits par l'auteur entre 2006 et 2012, publiés en italien et en anglais. Il a été présenté lors d'une séance du séminaire « Récits, valeurs et régimes de croyance », le 12/12/2017 à Metz. Je remercie A. Petitjean pour sa relecture attentive.

2. Les approches d'inspiration cognitiviste du hasard, comme celle de G. Bronner (2007), présentent les mêmes points de force et de faiblesse que celles de la philosophie analytique: on reconnait l'existence de vrai hasard dans le monde et on cherche à expliquer les interprétations "narratives» que les êtres humains en font comme des erreurs de raisonnement. On perd entièrement, cependant, la spécificité du rôle des coïncidences dans les récits que nous produisons pour donner un sens à la vie, par exemple dans la constitution de notre identité (Ricœur, 1988).

3. Au contraire, pour J. Searle (1975) les actions racontées ne suivent pas une grammaire spéciale : les jeux linguistiques seraient les mêmes dans la fiction comme dans la vraie vie.

4. C.S. Peirce, l'autre grand élève d'I. Kant, ne serait évidemment pas d'accord avec A. Schopenhauer: pour le philosophe américain nous n'avons aucun accès direct au noumène ou objet dynamique. U. Eco (2001) offre une médiation entre les deux penseurs à travers le concept d'iconisme primaire, qui nous relie directement - mais partiellement, parce que juste de façon négative - à l'objet $a$ quo, tel qu'il existe avant sa mise en forme sémiotique. Une discussion détaillée va au-delà des limites de cet essai.

5. Précisons que la même duplicité se trouve déjà dans des récits traditionnels (pas interactifs), mais dans les jeux vidéo elle devient plus évidente (Compagno, 2012).

6. Voir également Maietti (2004) pour un développement analogue.

7. Ces réactions sont rapportées par U. Eco dans les introductions des éditions successives de L'Euvre ouverte.

8. C'est à partir du concept d'œuvre en mouvement d'U. Eco qu'E. Aarseth (1997) et J. Murray (1997), indépendamment l'un de l'autre, ont parlé plus tard de littérature ergodique et de procéduralité, fondant les game studies. 


\section{RÉSUMÉS}

Les jeux et spécialement les jeux vidéo nous permettent de vivre une expérience impossible à réaliser autrement: celle du choix. Afin de comprendre la portée de cette considération, nous comparerons le modèle de l'action développé par E. Anscombe à celui de l'action racontée développé par A.J.Greimas, en montrant leur incompatibilité et leur insuffisance face aux actions interactives des jeux vidéo. Nous introduirons alors deux pensées capables d'enrichir notre compréhension. D'abord, celle d'A. Schopenhauer, que nous mobilisons pour décrire la nature hybride de l'avatar, à la foi personnage fictif et véhicule de la volonté réelle du joueur. Ensuite, la pensée d'U. Eco qui, à travers le concept d'ouverture, va permettre de caractériser l'expérience de liberté et de responsabilité associée à l'action interactive.

Games and especially video games allow us to live an experience that is impossible to live otherwise: the experience of choosing. In order to understand the consequences of such consideration we begin by comparing the model of action developed by E. Anscombe to the model of narrative action developed by A. J. Greimas. We show the incompatibility of these two models and their incapacity to account for interactive actions in video games. We then introduce two other perspectives capable of enriching our understanding. First, that of A. Schopenhauer, with whom we describe the hybrid nature of the avatar, at the same time fictional character and vehicle for the player's real will. Second, we recall U. Eco's concept of openness to characterize the experience of freedom and responsibility associated with interactive action.

\section{AUTEUR}

\section{DARIO COMPAGNO}

Université de Lorraine, Crem, F-57000 Metz, France 